\title{
Uncertain Volatility Derivative Model Based on the Polynomial Chaos
}

\author{
Stefanos Drakos \\ International Centre for Computational Engineering, Rhodes, Greece \\ Email: stefanos.drakos@gmail.com
}

Received 5 November 2015; accepted 16 February 2016; published 19 February 2016

Copyright (C) 2016 by author and Scientific Research Publishing Inc.

This work is licensed under the Creative Commons Attribution International License (CC BY).

http://creativecommons.org/licenses/by/4.0/

(c) (i) Open Access

\begin{abstract}
In the modern financial market the derivative pricing considers the use of historical or implied volatility which is actually the forward expectation of uncertainty. The common way of derivative pricing is to use the volatility as constant value in the well known Black Sholes equation. The aim of the current work was to develop a model where the uncertainty of volatility propagates to the derivative pricing and hedging according to the Black-Sholes PDE considering the volatility as stochastic process rather as a constant. A stochastic finite element method using generalized polynomial chaos was used to develop an algorithm of uncertainty propagation solving finally a deterministic problem for derivative pricing. The output of the method leads to derivative price distribution and the results of Monte Carlo Method for the derivative's distribution were used as the exact solution against those rose from the new algorithm.
\end{abstract}

\section{Keywords}

Uncertain Volatility Model, Polynomial Chaos, Quantification of Uncertainty, Stochastic Finite Element Method

\section{Introduction}

Several works were presented in the past for uncertainty quantification of derivative pricing due to random volatility. In [1] a new model for pricing and hedging derivative securities and option portfolios in an environment where the volatility is unknown and assumed to be ranged between two extreme values $\sigma_{\min }$ and $\sigma_{\max }$ was presented. The bound of volatility is computed by historical high-low peak of stock or option-implied volatilities and works as confidence interval for future volatility values. The derivative asset which arises as the volatility paths varies in such a band can be described by a non-linear PDE, which we call the Black-Scholes-Barenblatt equation. In [2] the pricing of contingent claims in a multidimensional frictionless security market assumed that the volatility of the security process is a known function of price and time. The author's approach was under- 
pinned by the theory of totally non-linear parabolic partial differential equations and the non-stochastic approach to Itô's formation. In [3] the volatility is considered as a random variable, which exhibits an adequate distribution like uniform type, Gaussian type or others. Thus the deterministic parabolic equation changes into a parabolic equation with stochastic input, where the solution represents a random field. Method of lines used for the numerical solution of the system resulting from the generalized polynomial chaos and corresponding endboundary value problems of a parabolic equation was solved numerically in a Monte Carlo simulation. In [4] the effect of uncertainty in the volatility parameter $\sigma$ on the Black-Scholes price of the European and American put was quantified. Probabilistic uncertainty analysis to the Black-Scholes model was carried out and the results are compared with those of the Uncertain Volatility model. The probability distribution for the volatility calibrated from historical data. The Monte Carlo (MC) and a surrogate Polynomial Chaos (PC)/MC methods were used to compute uncertainty bounds. The effect of the parametric uncertainty is investigated at the end.

In the current work a robust algorithm based on the stochastic finite element method using the generalized polynomial chaos was developed and it is considered as a general method for derivative pricing where the volatility input is considered as a random variable.

\section{Finite Element Formulation of Black-Sholes Equation}

Suppose $(\Omega, \mathcal{F}, \mathbb{P})$ is a probability space with a filtration $\left(\mathcal{F}_{t}\right)_{t \geq 0}$. Where $\mathcal{F}$ is the $\sigma$-algebra and is considered to contain all the information that is available, $\mathbb{P}$ is the probability measure. Consider a market consists of two assets, a risk free bond of constant interest rate $r$ and a stock with a price process $S_{t}$ evolving according to a geometric Brownian. The dynamic of assets are given by:

$$
\begin{gathered}
\mathrm{d} B(t)=r B(t) \mathrm{d} t \\
\mathrm{~d} S(t)=S(t) \mu \mathrm{d} t+S(t) \sigma \mathrm{d} W(t)
\end{gathered}
$$

where the drift rate $\mu$ and the volatility $\sigma>0$ are assumed to be constant, and $W(t)$ is a standard Brownian motion. The aim is to price a derivative of the form $\mathcal{X}=\Phi(S(T))$ under uncertain volatility. In the basic theory of BlackSholes the volatility of risky asset assumed to be constant which is results in mispricing of contingent claims due to uncertainty on the choice of the values within this model. As discussed in the previous paragraph in the current work the volatility considered as a stochastic process $\left\{\sigma(S, t, \omega): \mathbb{R}_{+} \in[0, T] \times \Omega\right\}$ following the uncertainty of the market prices. The distribution of volatility assumed to be lognormal and if the price of the derivative has the form $\mathcal{H}(t)=V(t, S(t), \omega)$ which is constintent with the absence of arbitrage then the function $V(t, S(t), \omega)$ is the solution of the following boundary value problem:

$$
\begin{cases}\frac{\partial V}{\partial t}(S, t, \omega)+\frac{1}{2} \sigma^{2} S^{2} \frac{\partial^{2} V}{\partial S^{2}}(S, t, \omega)+r S \frac{\partial V}{\partial S}(S, t, \omega)-r V(S, t, \omega)=0 & \text { in } \mathbb{R}_{+} \times[0, T] \times \Omega, \\ V(T, s)=\Phi(S(T)) & \text { in } \mathbb{R} .\end{cases}
$$

In essence the solution of the problem is a function of the form $u_{t} \in \mathbb{R} \times \Omega \rightarrow \mathbb{R}$ for every fixed $t$, i.e. a stochastic process and is not a deterministic function.

To solve the problem, we switch to the log-price process $X(t)=\log (S(t))$ which is the solution of the equation:

$$
\mathrm{d} X(t)=\left(r-\frac{1}{2} \sigma^{2}\right) \mathrm{d} t+\sigma \mathrm{d} W(t) .
$$

The infinitesimal generator for this process has constant coefficients:

$$
\mathcal{A}^{B S}=\frac{1}{2} \sigma^{2} \partial_{x x}+\left(r-\frac{1}{2} \sigma^{2}\right) \partial_{x} .
$$

Thus by setting $V(t, S, \omega)=u(T-t, \log S, \omega)$ the Equation (3) becomes:

$$
\left\{\begin{array}{l}
\frac{\partial u}{\partial t}-\mathcal{A}^{B S} u+r u=0 \quad \text { in } \mathbb{R}_{+} \times[0, T] \times \Omega \\
V(0, x)=\Phi\left(\mathrm{e}^{x}\right) \text { in } \mathbb{R} .
\end{array}\right.
$$

In order to solve the problem according to the finite element method in the current paper we consider a linear 
element with nodes $N_{i}\left(x^{(i)}\right), i=1,2$. To each node $N_{i}$ there is a hat function $\varphi_{i}$. To solve the problem assuming a test $v$ function belongs to the space:

$$
V^{h}=\operatorname{span}\left\{\varphi_{1}, \varphi_{2}, \cdots, \varphi_{N}\right\} \subset H_{0}^{1}(D) .
$$

Using a test function $v$ and integrating by parts over the domain $D$ the variational formulation of the BlackScholes equation has the following form:

$$
\left\{\begin{array}{l}
\left(\partial u_{t}, v\right)+\frac{1}{2} \sigma^{2}\left(\partial u_{x}, \partial v_{x}\right)+\left(\frac{1}{2} \sigma^{2}-r\right)\left(\partial u_{x}, v\right)+r(u, v)=0, \\
u(0)=\Phi\left(\mathrm{e}^{x}\right) .
\end{array}\right.
$$

Using the matrix notation the equation takes the following form:

$$
\left\{\begin{array}{l}
\frac{d u}{\Delta t} \boldsymbol{M}+\frac{1}{2} \sigma^{2}(\boldsymbol{K}+\boldsymbol{C}) \cdot u+r(\boldsymbol{M}-\boldsymbol{C}) \cdot u=0 \\
u(0)=\Phi\left(\mathrm{e}^{x}\right)
\end{array}\right.
$$

where:

$$
\left\{\begin{array}{l}
\boldsymbol{M}=\int_{D} \varphi_{i}(\boldsymbol{x}) \varphi_{j}(\boldsymbol{x}) \mathrm{d} x, \\
\boldsymbol{K}=\int_{D} \nabla \varphi_{i}(\boldsymbol{x}) \nabla \varphi_{j}(\boldsymbol{x}) \mathrm{d} x, \\
\boldsymbol{C}=\int_{D} \nabla \varphi_{i}(\boldsymbol{x}) \varphi_{j}(\boldsymbol{x}) \mathrm{d} x .
\end{array}\right.
$$

\section{Stochastic Galerkin Solution}

Assuming that the volatility of stock $\boldsymbol{\sigma}(\boldsymbol{t}, \boldsymbol{x}, \boldsymbol{\xi}(\omega))$ for $\boldsymbol{t} \in[0, T], \boldsymbol{x} \in D$ and $\omega \in \Omega$ dependent of a random variable $\xi: \Omega \rightarrow \Gamma \subset \mathbb{R}$. To compute the statistical moments of the problem we perform a change of variable $y:=\xi(\omega)$. The calculating procedure for the expected value using instead of the abstract space $\Omega$ of random variable $\xi$ its figure leads to solve a deterministic problem in space $D \times \Gamma \subset \mathbb{R}$ instead of space $D \times \Omega$. By performing such replacements in fact a deterministic problem is solved, in contrast to the case of Monte Carlo where a large number of problems carried out.

The author has presented a stochastic finite element procedure to solve boundary problems using polynomial chaos [5]-[9]. The outcome derivative price of the problem is given by the polynomial chaos expansion as:

$$
u(\boldsymbol{t}, \boldsymbol{x}, \boldsymbol{y})=\sum_{k=1}^{Q} u_{k}(\boldsymbol{t}, \boldsymbol{x}) \psi_{\kappa}(\boldsymbol{y})
$$

where the order $Q$ and the formula $\psi$ of Polynomial Chaos are given in Appendix.

According that and using the inner product of the equation on each polynomial of the $S^{k}$ base we get:

$$
\left\langle\sum_{k=1}^{Q} \frac{d u_{k}(\boldsymbol{t}, \boldsymbol{x})}{\Delta t} \psi_{\kappa}(\boldsymbol{y}) \boldsymbol{M}+\frac{1}{2} \sigma^{2}(\boldsymbol{K}+\boldsymbol{C}) \cdot \sum_{k=1}^{Q} u_{k}(\boldsymbol{t}, \boldsymbol{x}) \psi_{\kappa}(\boldsymbol{y})+r(\boldsymbol{M}-\boldsymbol{C}) \cdot \sum_{k=1}^{Q} u_{k}(\boldsymbol{t}, \boldsymbol{x}) \psi_{\kappa}(\boldsymbol{y}), \psi_{p}\right\rangle=0 .
$$

Making the replacement of matrices and assuming $\rho(\boldsymbol{y})$ the density function we take the equation:

$$
\begin{aligned}
& \sum_{i}^{\text {nnode }} \sum_{k}^{Q} \frac{\partial u_{i k}(\boldsymbol{t}, \boldsymbol{x})}{\partial t} \int_{\Gamma} \rho(\boldsymbol{y}) \psi_{k}(\boldsymbol{y}) \psi_{p}(\boldsymbol{y}) \int_{D} \varphi_{i}(\boldsymbol{x}) \cdot \varphi_{j}(\boldsymbol{x}) \mathrm{d} x \mathrm{~d} y \\
& +\sum_{i}^{\text {nnode }} \sum_{k}^{Q} \frac{\partial u_{i k}(\boldsymbol{t}, \boldsymbol{x})}{\partial t} \int_{\Gamma} \rho(\boldsymbol{y}) \psi_{k}(\boldsymbol{y}) \psi_{p}(\boldsymbol{y}) \int_{D} \varphi_{i}(\boldsymbol{x}) \cdot \varphi_{j}(\boldsymbol{x}) \mathrm{d} x \mathrm{~d} y \\
& +\frac{1}{2} \mathrm{e}^{2 \mu_{l n}} \sum_{i}^{\text {nnode }} \sum_{k}^{Q} u_{i k}(\boldsymbol{t}, \boldsymbol{x}) \int_{\Gamma} \rho(\boldsymbol{y}) \psi_{k}(\boldsymbol{y}) \psi_{p}(\boldsymbol{y}) \mathrm{e}^{2 \sigma_{l n} \boldsymbol{y}}\left(\int_{D} \nabla \varphi_{i}(\boldsymbol{x}) \cdot \nabla \varphi_{j}(\boldsymbol{x}) \mathrm{d} x+\int_{D} \nabla \varphi_{i}(\boldsymbol{x}) \cdot \varphi_{j}(\boldsymbol{x}) \mathrm{d} x\right) \mathrm{d} y \\
& +r \sum_{i}^{\text {nnode }} \sum_{k}^{Q} u_{i k}(\boldsymbol{t}, \boldsymbol{x}) \int_{\Gamma} \rho(\boldsymbol{y}) \psi_{k}(\boldsymbol{y}) \psi_{p}(\boldsymbol{y})\left(\int_{D} \varphi_{i}(\boldsymbol{x}) \cdot \varphi_{j}(\boldsymbol{x}) \mathrm{d} x \int_{D} \nabla \varphi_{i}(\boldsymbol{x}) \cdot \varphi_{j}(\boldsymbol{x}) \mathrm{d} x\right) \mathrm{d} y=0 .
\end{aligned}
$$


This is equivalent with:

$$
\begin{aligned}
& \frac{\mathrm{d} u}{\mathrm{~d} t} \boldsymbol{M} \otimes\left\langle\psi_{k}(\boldsymbol{y}) \psi_{p}(\boldsymbol{y})\right\rangle \cdot u(\boldsymbol{t}, \boldsymbol{x})+\frac{1}{2} \mathrm{e}^{2 \mu_{l n}}(\boldsymbol{K}+\boldsymbol{C}) \otimes\left\langle\psi_{k}(\boldsymbol{y}) \mathrm{e}^{2 \sigma_{l n} y} \psi_{p}(\boldsymbol{y})\right\rangle \cdot u(\boldsymbol{t}, \boldsymbol{x}) \\
& +r(\boldsymbol{M}-\boldsymbol{C}) \otimes\left\langle\psi_{k}(\boldsymbol{y}) \psi_{p}(\boldsymbol{y})\right\rangle \cdot u(\boldsymbol{t}, \boldsymbol{x})=0 .
\end{aligned}
$$

To simplify the form make the following:

$$
\left\{\begin{array}{l}
\boldsymbol{M P}=\boldsymbol{M} \otimes\left\langle\psi_{k}(\boldsymbol{y}) \psi_{p}(\boldsymbol{y})\right\rangle, \\
\mathcal{A}^{B S P}=\frac{1}{2} \mathrm{e}^{2 \mu_{l n}}(\boldsymbol{K}+\boldsymbol{C}) \otimes\left\langle\psi_{k}(\boldsymbol{y}) \mathrm{e}^{2 \sigma_{l n} \boldsymbol{y}} \psi_{p}(\boldsymbol{y})\right\rangle+r(\boldsymbol{M}-\boldsymbol{C}) \otimes\left\langle\psi_{k}(\boldsymbol{y}) \psi_{p}(\boldsymbol{y})\right\rangle .
\end{array}\right.
$$

\section{Time Discretization}

According to the previous replacement we discretize the Equation (14) using the theta-scheme with constant time step $\Delta t$. The finite element mesh considered as uniform and the Equation (14) takes the following form:

$$
\boldsymbol{M P}+\theta \mathcal{A}^{B S P} u^{n+1}+(1-\theta) \mathcal{A}^{B S P} u^{n}=0 .
$$

Equivalent

$$
\left(\boldsymbol{M P}+\theta \mathcal{A}^{B S P}\right) u^{n+1}=\left(\boldsymbol{M P}-(1-\theta) \mathcal{A}^{B S P}\right) u^{n} .
$$

The statistical moments of the outcome derivative price arise by the properties of the Polynomial of Chaos expansion:

The expected value:

$$
\mathbb{E}[u(\boldsymbol{t}, \boldsymbol{x}, \boldsymbol{y})]=\mathbb{E}\left[\sum_{k=0}^{P} u_{k}(\boldsymbol{t}, \boldsymbol{x}) \psi_{\kappa}(\boldsymbol{y})\right]=u_{0}(\boldsymbol{t}, \boldsymbol{x}) \underbrace{\mathbb{E}\left[\psi_{0}(\boldsymbol{y})\right]}_{1}+\underbrace{\sum_{k=1}^{K} u_{k}(\boldsymbol{t}, \boldsymbol{x}) \mathbb{E}\left[\psi_{\kappa}(\boldsymbol{y})\right]}_{0}=u_{0}(\boldsymbol{t}, \boldsymbol{x})
$$

and the variance:

$$
\begin{aligned}
& \sigma^{2}=\mathbb{E}(u(\boldsymbol{t}, \boldsymbol{x}, \boldsymbol{y})-\mathbb{E}[u(\boldsymbol{t}, \boldsymbol{x}, \boldsymbol{y})])^{2}=\mathbb{E}\left(\sum_{k=0}^{K} u_{k}(\boldsymbol{t}, \boldsymbol{x}) \mathbb{E}\left[\psi_{\kappa}(\boldsymbol{y})\right]-u_{0}(\boldsymbol{t}, \boldsymbol{x})\right) \\
& \Rightarrow \sigma^{2}=\sum_{k=0}^{K} u_{k}^{2}(\boldsymbol{t}, \boldsymbol{x}) \mathbb{E}\left[\psi_{\kappa}^{2}(\boldsymbol{y})\right] .
\end{aligned}
$$

\section{Numerical Example}

For the numerical example of derivative pricing a plain vanilla European Put Option was chosen. The historical data of S\&P 500 was used to compute the mean value and the volatility of the index volatility. Based on the mean and max value of volatility and its mean volatility of volatility the option price has been calculated with expiration time $T=90 / 360$ and strike price $K=1200$. The statistical value of historical data for volatility for a period from 2000-2014 are given in Table 1. In Figure 1 and Figure 2 the Daily Return and the volatility of S\&P 500 are presented.

To verify the model we compare the results with those raised by the Monte Carlo method which is treated as the exact solution. The computational implementation of the Monte Carlo Method leads to the random process generation of $\sigma_{k}$ and the requested $V(t, S)$ gets a new value $\left\{V_{k}(S)\right\}_{k}^{K}$ for each realization, where $V_{k}(S)=V\left(S, \sigma_{k}\right)$. At the end of all simulations the statistical moment are calculated.

The expected value and the variance are given by:

$$
\left\{\begin{array}{l}
\mathbb{E}(V(t, S))=\frac{1}{K} \sum_{k=1}^{K} V_{k}(S), \\
\operatorname{Var}(V(t, S))=\frac{1}{K-1} \sum_{k=1}^{K}\left(V_{k}(S)\right)-\mathbb{E}\left(V_{k}(S)\right)^{2} .
\end{array}\right.
$$




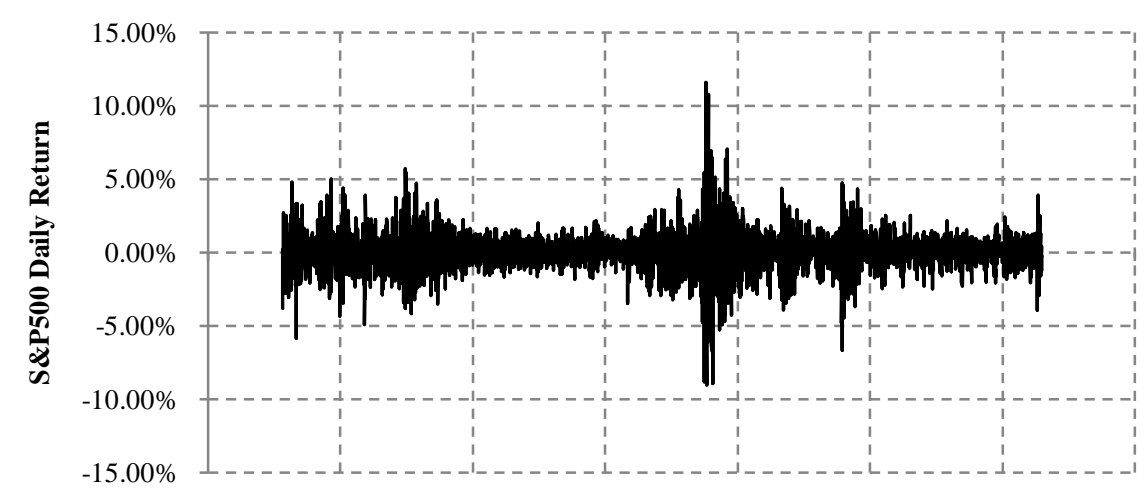

24/7/1998 19/4/2001 14/1/2004 10/10/2006 6/7/2009 1/4/2012 27/12/2014 22/9/2017

Figure 1. Daily returns of S\&P 500 for the period 2000-2014 (Yahoo finance).

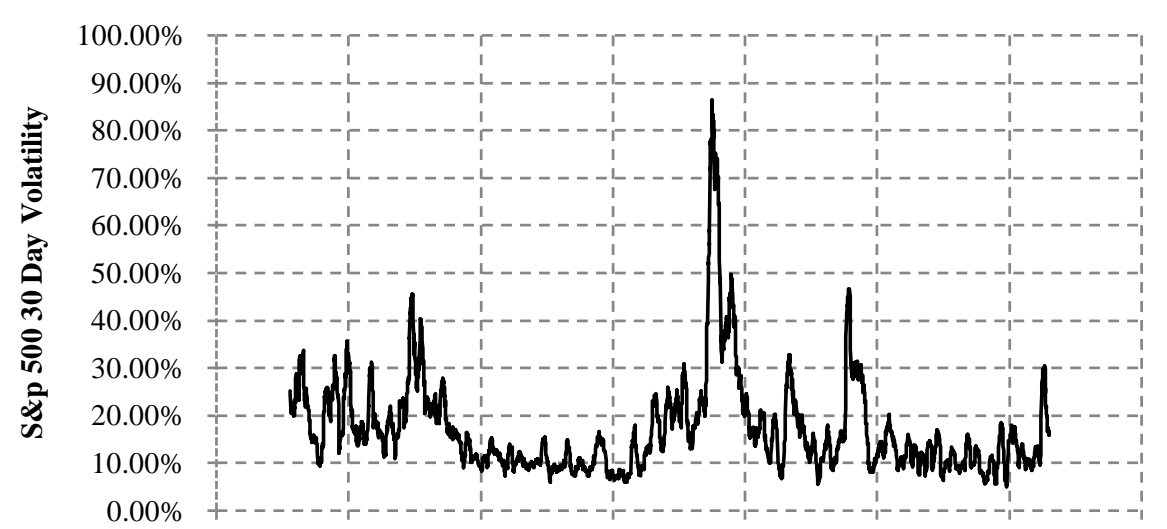

24/7/1998 19/4/2001 14/1/200410/10/2006 6/7/2009 1/4/2012 27/12/201422/9/2017

Figure 2. 30 day volatility of S\&P 500 for the period 2000-2014 (Yahoo finance).

Table 1. Statistical values of volatility of S\&P 500 for the period 2000-2014.

\begin{tabular}{cccc}
\hline & \multicolumn{2}{c}{ Volatility of S\&P $\mathbf{5 0 0}$} & $\mathbf{2 0 0 0 - 2 0 1 4}$ \\
\hline Min & Mean & Max & Vol of Vol \\
$5.01 \%$ & $17.20 \%$ & $86.35 \%$ & $10.58 \%$ \\
\hline
\end{tabular}

Two different cases were carried out (Table 2).

In the Figure 3 and Figure 4 the results of the statistical moments of the derivative pricing are presented and high accuracy is realized. The effect of volatility uncertainty on the statistical moments of option price presented in Figure 5 and Figure 6. For 10 values of index volatility of volatility with a mean value equal to $40 \%$ and input parameters as before, 10 different calculations were carried out and the results are presented. The increase of vol of vol causes a decrease in expected option price of $12 \%$ approximately at the maxim while results a huge increment on its volatility as presented in Figure 6.

\section{Conclusion}

A new algorithm of volatility uncertainty propagation in the derivative pricing and hedging procedure was presented according to the Black-Sholes PDE. A stochastic finite element method using generalized polynomial chaos was used to develop an algorithm of uncertainty propagation solving finally a deterministic problem for the derivative pricing. The results of Monte Carlo Method for the derivative's distribution were used as the exact solution against those rose from the new algorithm. The method leads to high accuracy and eliminates the large number of the Monte Carlo Method's simulations. The model applied using the historical data of S\&P 500 for 
Table 2. Cases of analysis.

\begin{tabular}{ccc}
\hline & Mean of volatility & Volatility of volatility \\
\hline Case I & $18.7 \%$ & $10.6 \%$ \\
Case II & $86.35 \%$ & $10.6 \%$ \\
\hline
\end{tabular}

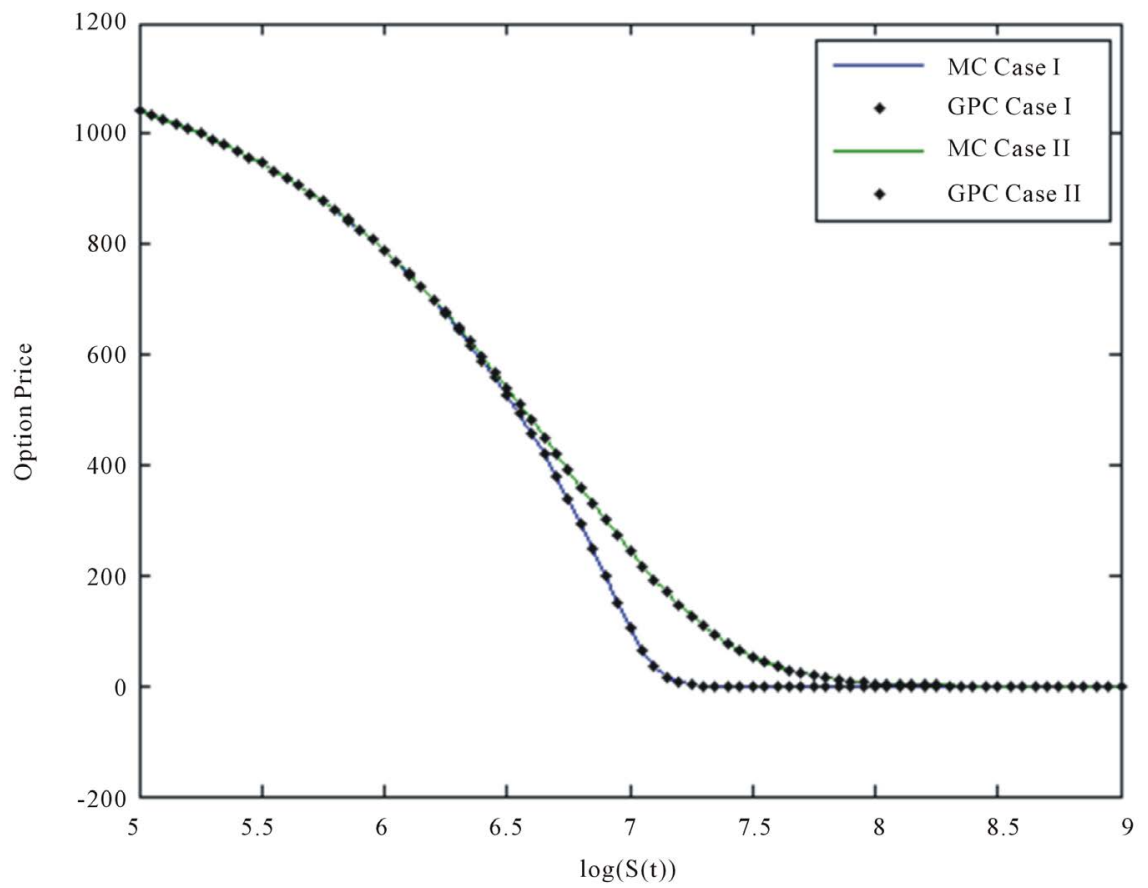

Figure 3. Option price expected value.

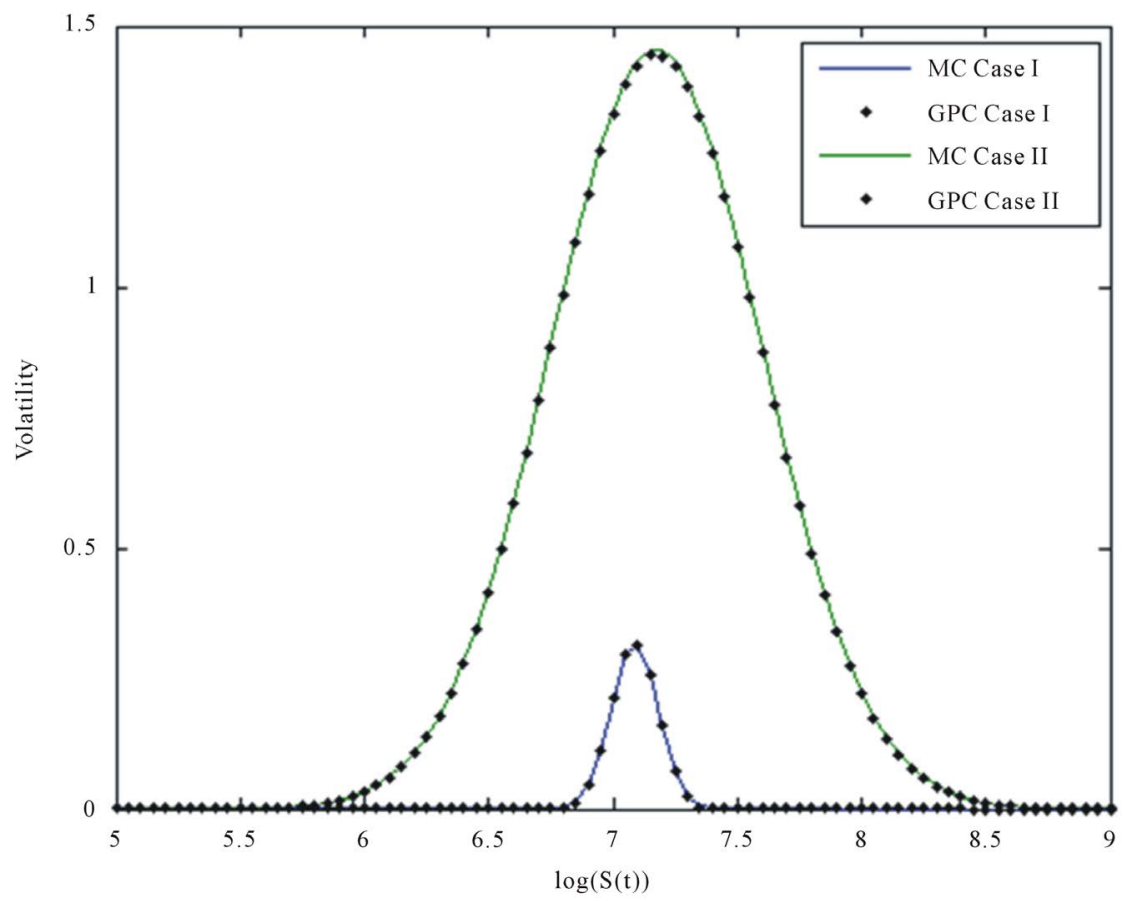

Figure 4. Volatility of option price. 


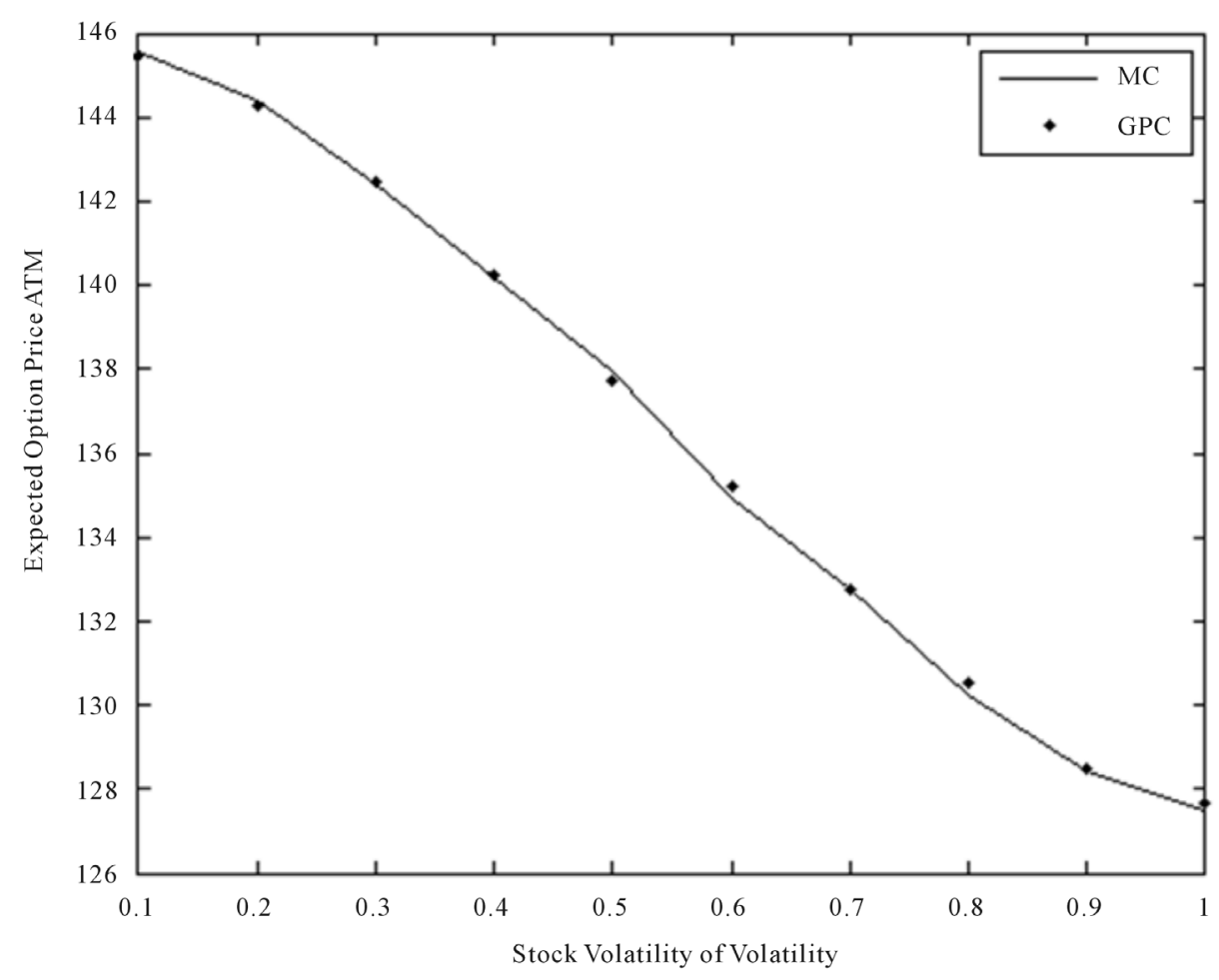

Figure 5. Expected option price ATM via stock volatility of volatility.

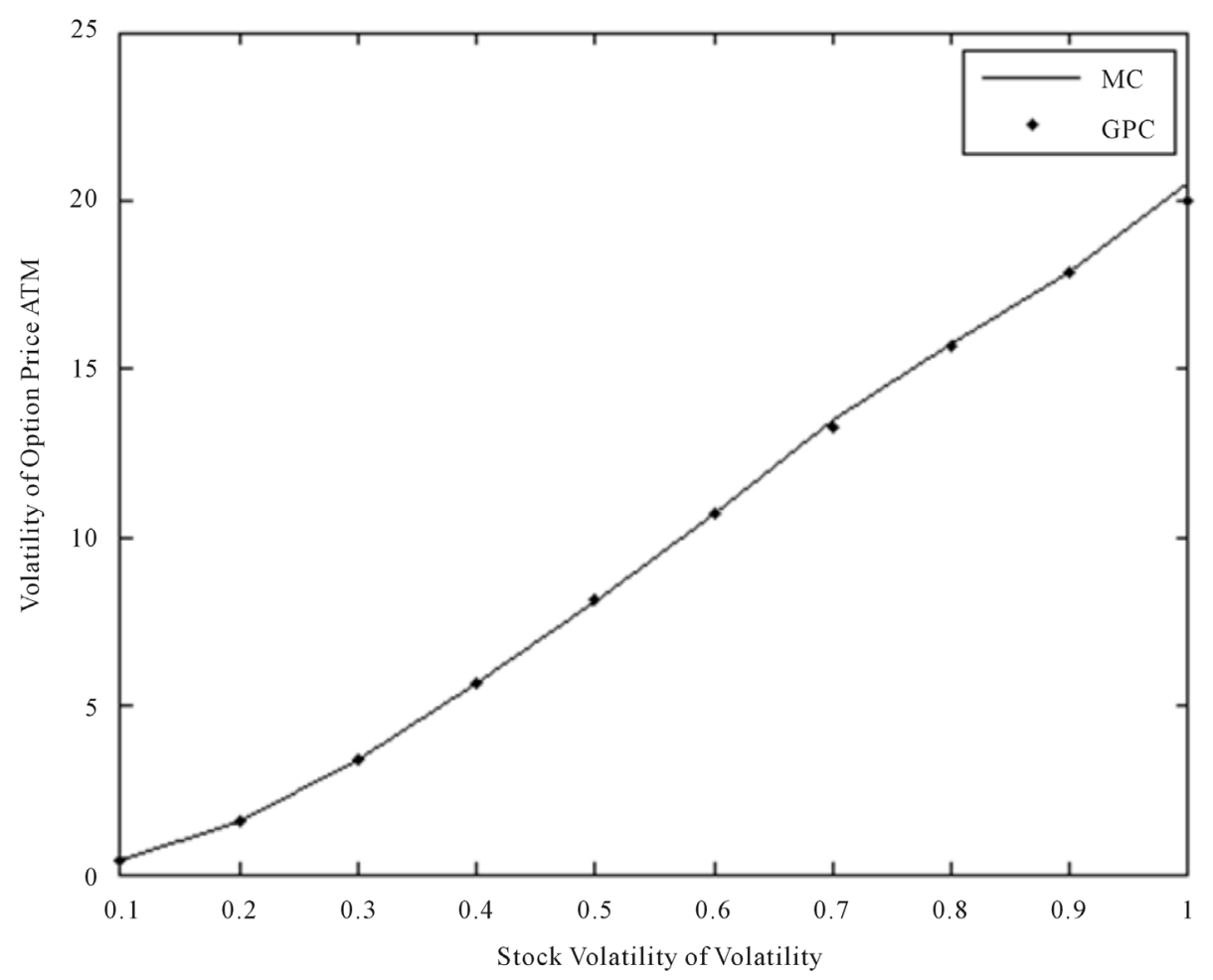

Figure 6. Volatility of option price ATM via stock volatility of volatility.

the period 2000-2014. The mean value and the volatility of volatility of index are used for the pricing of a plain vanilla European put option considering the volatility parameter as a stochastic process. The effect of index vo- 
latility of volatility on the derivative price distribution was analyzed and the results were presented by the two methods. High uncertainty of index volatility leads to a highly non linear increase of option price volatility as the results of analysis are shown.

\section{References}

[1] Avellaneda, M., Levy, A. and Para, A. (1995) Pricing and Hedging Derivative Securities in Markets with Uncertain Volatilities. Applied Mathematical Finance, 2, 73-88. http://dx.doi.org/10.1080/13504869500000005

[2] Lyons, T.J. (1995) Uncertain Volatility and the Risk-Free Synthesis of Derivatives. Applied Mathematical Finance, 2, 117-133. http://dx.doi.org/10.1080/13504869500000007

[3] Pulch, R. and van Emmerich, C. (2009) Polynomial Chaos for Simulating Random Volatilities. Mathematics and Computers in Simulation, 80, 245-255. http://dx.doi.org/10.1016/j.matcom.2009.05.008

[4] Namihira, M. and Kopriva, D.A. (2012) Computation of the Effects of Uncertainty in Volatility on Option Pricing and Hedging. International Journal of Computer Mathematics, 89, 1281-1302. http://dx.doi.org/10.1080/00207160.2012.688819

[5] Drakos, I.S. (2015) Elastic Stress Predictor for Stochastic Finite Element Problems. World Journal of Mechanics, 5, 222-233. http://dx.doi.org/10.4236/wjm.2015.511021

[6] Drakos, I.S. and Pande, G.N. (2015) Stochastic Finite Element Analysis for Transport Phenomena in Geomechanics Using Polynomial Chaos. Civil and Structural Engineering, 15.

[7] Drakos, I.S. and Pande, G.N. (2015) Quantitative of Uncertainties in Earth Structures. World Congress on Advances in Structural Engineering and Mechanics, Incheon, 25-29.

[8] Drakos, I.S. (2015) Quantitative of Uncertainty in Unconfined Flow Problems. International Journal of Geotechnical Engineering, Accepted for Publishing.

[9] Drakos, S.I. (2015) Constitutive Relations of Stress and Strain in Stochastic Finite Element Method. American Journal of Computational and Applied Mathematics, 5, 164-173.

[10] Lord, G., Powel, C. and Shardlow, T. (2014) An Introduction to Computational Stochastic PDEs. Cambridge Texts in Applied Mathematics. http://dx.doi.org/10.1017/CBO9781139017329

[11] Xiu, D. and Em Karniadakis, G. (2003) Modeling Uncertainty in Steady State Diffusion Problems via Generalized Polynomial Chaos. Computer Methods in Applied Mechanics and Engineering, 191, 4927-4948. http://dx.doi.org/10.1016/S0045-7825(02)00421-8 


\section{Appendix}

\section{Galerkin Approximation and Generalized Polynomial of Chaos}

In order to solve the problem 3 we have to create the new space $L_{p}^{2}\left(\Gamma, H_{0}^{1}(D)\right)$. For that reason the subspace $S^{k} \subset L_{p}^{2}(\Gamma)$ is considered as [10].

$$
S^{k}=\operatorname{span}\left\{\psi_{1}, \psi_{2}, \cdots, \psi_{\kappa}\right\} .
$$

Using the dyadic product of the space $V^{h}, S^{k}$ the space $L_{p}^{2}\left(\Gamma, H_{0}^{1}(D)\right)$ created. Thus

$$
V^{h k}=V^{h} \otimes V^{k}=\operatorname{span}\left\{\varphi_{i} \psi_{j}, i=1, \cdots, N, j=1, \cdots, Q\right\} .
$$

The space $V^{h k}$ has dimension QN and regards the test function $v$. In the case where exists $N_{B}$ finite element supported by boundaries condition then the subspace of solution belongs is:

$$
W^{h k}=V^{h k} \oplus \operatorname{span}\left\{\varphi_{N+1}, \varphi_{N+2}, \cdots, \varphi_{N+N B}\right\} .
$$

Assuming that the $S_{i}^{k}$ represents a space of univariate orthonormal polynomial of variable $y_{i} \subset \Gamma_{\iota} \subset \mathbb{R}$ with order $k$ or lower and:

$$
S_{i}^{k}=\operatorname{span}\left\{P_{a_{i}}^{i}\left(y_{i}\right), a_{i}=0,1,2, \cdots, k\right\}, i=1, \cdots, M .
$$

The tensor product of the $M S_{i}^{k}$ subspace results the space of the Generalized Polynomial Chaos:

$$
S^{k}=S_{1} \otimes S_{2} \otimes \cdots \otimes S_{M} .
$$

And using (A4)

$$
S^{k}=\operatorname{span}\left\{\prod_{i=1}^{M} P_{a_{i}}^{i}\left(y_{i}\right): a_{i}=0,1, \cdots, k, i=1, \cdots, M,|a| \leq k\right\}
$$

where $|a|=\sum_{i=1}^{M} a_{i}$.

And

$$
Q=\operatorname{dim}\left(S^{k}\right)=\frac{(M+k) !}{M ! k !} .
$$

Xiu \& Karniadakis [11] show the application of the method for different kind of orthonormal polynomials and in the current paper the Hermite polynomial was used with the following characteristics:

$$
\begin{gathered}
P_{0}=1,\left\langle P_{i}\right\rangle=0, i>0 \\
\left\langle P_{m} P_{n}\right\rangle=\int_{\Gamma} P_{m}(\boldsymbol{y}) P_{n}(\boldsymbol{y}) \rho(\boldsymbol{y}) \mathrm{d} \boldsymbol{y}=\gamma_{n} \delta_{m n}
\end{gathered}
$$

where:

$\gamma_{n}=\left\langle P_{n}^{2}\right\rangle$ : are the normalization factors, $\delta_{m n}$ is the Kronecker delta.

$\rho(y)=\frac{1}{\sqrt{2 \pi}} \mathrm{e}^{-\frac{y}{2}}:$ is the density function and

$$
P_{n}=(-1)^{n} \mathrm{e}^{\frac{y}{2}} \frac{\mathrm{d}^{n}}{\mathrm{~d} y^{n}} \mathrm{e}^{-\frac{y}{2}}
$$

For a $3^{\text {rd }}$ order of one dimension of uncertainty the Hermite Polynomial Chaos is given by: $\psi_{o}(y)=P_{0}(y)=1, \quad \psi_{1}(y)=P_{1}(y)=y, \quad \psi_{2}(y)=P_{2}(y)=y^{2}-1$. 